\title{
EXPANSÃO DOS CURSOS DE GRADUAÇÃO EM ENFERMAGEM NO BRASIL ENTRE 2004 E 2017
}

Leticia Katiane Martins ${ }^{1}$

Rosa Maria Rodrigues ${ }^{1}$

Raphael Klein de Souza ${ }^{2}$

Solange de Fátima Reis Conterno ${ }^{1}$

Mateus Souza da Luz https://orcid.org/0000-0002-8484-6040 https://orcid.org/0000-0002-7047-037X https://orcid.org/0000-0002-6896-4135 https://orcid.org/0000-0003-2493-8071

https://orcid.org/0000-0003-2405-0152

Resumo: Objetivou-se analisar o desenvolvimento e a expansão dos cursos de graduação em enfermagem no Brasil no período de 2004 a 2017. Estudo exploratório, de caráter quantitativo através de dados documentais disponibilizados pelo Inep. A expansão se deu primordialmente pela esfera privada com a região Sudeste responsável pela maioria dos cursos. A expansão foi estimulada por programas federais de apoio ao acesso e permanência. Houve ampliação de cursos nas regiões Norte e Nordeste, o que não se observa no restante do território brasileiro, onde há desaceleração da expansão e extinção de cursos. Identificou-se a educação a distância como realidade na área. Conclui-se que permanece a expansão de cursos majoritariamente privados, com tendência a desaceleração, e extinção nas regiões Sul e Sudeste.

Descritores: Educação; Educação Superior; Enfermagem

\section{EXPANSION OF GRADUATION COURSES IN NURSING IN BRAZIL BETWEEN 2004 AND 2017}

Abstract: The objective of this study was to analyze the development and expansion of undergraduate nursing courses in Brazil from 2004 to 2017. This is an exploratory study of quantitative nature through documentary data provided by Inep. The expansion took place primarily in the private sphere with the Southeast region responsible for most courses. The expansion was stimulated by federal programs to support access and permanence. There has been expansion of courses in the North and Northeast regions, which is not observed in the rest of Brazil, where there is a slowdown in the expansion and extinction of courses. Distance education was identified as a reality in the area. It is concluded that the expansion of courses mostly private, with a tendency to deceleration, and extinction in the South and Southeast regions.

Descriptors: Education; College education; Nursing.

\section{EXPANSIÓN DE LOS CURSOS DE GRADUACIÓN EN ENFERMERÍA EN BRASIL ENTRE 2004 Y 2017}

Resumen: Se objetivó analizar el desarrollo y la expansión de los cursos de graduación en enfermería en Brasil en el período de 2004 a 2017. Estudio exploratorio, de carácter cuantitativo a través de datos documentales disponibilizados por el Inep. La expansión se dio primordialmente por la esfera privada con la región Sudeste responsable de la mayoría de los cursos. La expansión fue estimulada por programas federales de apoyo al acceso y permanencia. Se ha ampliado los cursos en las regiones Norte y Nordeste, lo que no se observa en el resto del territorio brasileño, donde hay desaceleración de la expansión y extinción de cursos. Se identificó la educación a distancia como realidad en el área. Se concluye que permanece la expansión de cursos mayoritariamente privados, con tendencia a desaceleración, y extinción en las regiones Sur y Sudeste.

Descriptores: La educación; Educación universitaria; Enfermeria

'Universidade Estadual do Oeste do Paraná (Unioeste). Cascavel, Paraná,

2Universidade Tecnológica Federal do Paraná - UTFPR. Toledo, Paraná

Autor correspondente: Letícia Katiane Martins - Email: E-mail: leticiakmartins2@gmail.com 


\section{INTRODUÇÃO}

Nos últimos anos houve aumento progressivo dos cursos, vagas e ingressantes em todas as áreas do conhecimento, inclusive na Enfermagem, principalmente na região Sudeste. Essa ampliação deveria ser acompanhada da discussão sobre o seu papel social e da força política para reduzir a precarização do mundo do trabalho(1). A expansão pode parecer positiva para a defesa da democratização do ensino superior, mas é preciso que seja pensada, planejada e regulada de acordo com as exigências do mercado de trabalho e de cada região, que é o destino dos futuros profissionais ${ }^{(2)}$.

Comprovando esse processo de expansão, observou-se que desde 1922 até 1964 abriram-se 39 cursos de enfermagem no Brasil. Em 1991 esse número passou para 106, em 2004, 415 e em 2011, eram 826 cursos $^{(3)}$.

Após esse crescimento, percebe-se empiricamente, que há um movimento de desaceleração da expansão dos cursos no estado do Paraná através da desativação de alguns cursos, o que pode estar ocorrendo em nível nacional. Assim, após a expansão dos cursos de enfermagem no Brasil, questiona-se como se encontra a distribuição dos cursos de enfermagem no Brasil, especialmente entre os anos de 2004 a 2017? O objetivo do estudo foi sistematizar e analisar o desenvolvimento e a expansão dos cursos de graduação em enfermagem no Brasil no período de 2004 a 2017.

\section{METODOLOGIA}

\section{Tipo de estudo}

Trata-se de um estudo exploratório, documental, de caráter quantitativo. Os dados foram acessados no banco de dados do censo da educação superior produzidos pelo Instituto Nacional de Estudos e Pesquisas Educacionais Anísio Teixeira (Inep).

\section{Fonte, procedimentos e período para coleta dos dados}

O acesso ao banco de dados completo com as informações sobre a evolução dos cursos de graduação em enfermagem foi realizado no ano de 2017, através de solicitação no Sistema Eletrônico do Serviço de Informação ao Cidadão disponível no endereço: <https://esic.cgu.gov.br/ sistema/site/index.aspx?ReturnUrl=\%2fsistema\%2fPedido\%2fDetalhePedido.aspx\%3fid\%3dJY8dPLsmCB0\%3dधi$d=J Y 8 d P L s m C B O=>^{(4)}$, no qual solicitou-se os dados exclusivos da graduação em enfermagem, que foram enviados em planilhas do Microsoft Excel, de forma que pudessem ser sistematizados. Os dados disponíveis e recebidos foram do ano de 1991 a 2016. Os dados de 2017 foram acessados por consulta direta ao sistema e-MEC do Ministério da Educação, em setembro de 2017 que disponibiliza estatísticas relativas a educação superior: <http://emec.mec.gov.br/>(5). Os dados utilizados são de domínio público, não necessitando de envio de projeto para apreciação ética, conforme Resolução CNS 510/2016.

\section{Análise dos dados}

Os dados foram sistematizados com auxílio do Microsoft Excel considerando as seguintes variáveis: localização regional, definição do curso, organização acadêmica, modalidade de ensino, formas de oferta. Foram distribuídos em tabelas e gráficos de frequências absolutas e relativas, analisados e discutidos baseando-se na produção teórica sobre educação superior e expansão do ensino em enfermagem no Brasil.

\section{RESULTADOS}

Estavam em atividade em 2016, 983 cursos de enfermagem e, em 2017, 1.256 cursos, dos quais, em 2016, quase a metade era da região Sudeste $(43,7 \%)$ (tabela 1). A região Nordeste se destaca pelo percentual de criação de cursos no período. A região Norte teve um crescimento de 57 cursos, correspondendo a uma variação de $271,4 \%$. Apesar deste percentual de variação importante, esta região responde pelo menor número de cursos no Brasil, em 2016.

A região Sudeste teve percentual de expansão baixo (95,5\%), em relação às demais regiões, porém em números absolutos, continua a responder pelo maior quantitativo, com 430 cursos no ano de 2016. A região contava com $43,19 \%$ dos cursos de enfermagem do país, sendo que 22,28\% localizavam-se em São Paulo.

Observa-se que, além de haver uma discrepância intra-regional, há uma desproporção no interior dos estados, evidenciado pelo percentual na capital de São Paulo, contemplando quase metade dos cursos.

Por fim, na região Sul, o estado que mais expandiu foi Rio Grande do Sul, enquanto o que menos expandiu foi Santa Catarina.

Em estados como Alagoas, Amapá e Roraima observam-se aumentos percentuais que podem não significar cobertura homogênea de cursos quando comparado a estados de outras regiões. Estima-se que essa característica seja resultado das diferenças sociais e econômicas e populacionais entre uma região e outra configurando a desigualdade na distribuição dos cursos no Brasil. 
Tabela 1. Relação entre os anos de 2004 e 2016 do total de cursos de graduação em enfermagem no Brasil, por Unidade Federativa.

\begin{tabular}{|c|c|c|c|c|c|c|c|}
\hline \multirow{2}{*}{$\begin{array}{l}\text { Região } \\
\text { Geográfica }\end{array}$} & \multirow{2}{*}{$\begin{array}{l}\text { Unidade } \\
\text { Federativa }\end{array}$} & \multicolumn{2}{|c|}{2004} & \multicolumn{2}{|c|}{2016} & \multicolumn{2}{|c|}{$\begin{array}{c}\text { Variação } \\
2004-2016\end{array}$} \\
\hline & & $\mathrm{N}$ & $\%$ & $\mathrm{~N}$ & $\%$ & $\mathrm{~N}$ & $\%$ \\
\hline \multirow[t]{10}{*}{ Nordeste } & Alagoas & 2 & 0,5 & 14 & 1,4 & 12 & 600,0 \\
\hline & Pernambuco & 7 & 1,7 & 29 & 3,0 & 22 & 314,3 \\
\hline & Ceará & 7 & 1,7 & 30 & 3,1 & 23 & 328,6 \\
\hline & Bahia & 15 & 3,6 & 66 & 6,7 & 51 & 340,0 \\
\hline & $\begin{array}{l}\text { Rio Grande do } \\
\text { Norte }\end{array}$ & 4 & 1,0 & 17 & 1,7 & 13 & 325,0 \\
\hline & Maranhão & 8 & 1,9 & 27 & 2,7 & 19 & 237,5 \\
\hline & Sergipe & 3 & 0,7 & 11 & 1,1 & 8 & 266,7 \\
\hline & Paraiba & 7 & 1,7 & 20 & 2,0 & 13 & 185,7 \\
\hline & Piauí & 8 & 1,9 & 21 & 2,1 & 13 & 162,5 \\
\hline & Subtotal & 61 & 14,7 & 235 & 23,9 & 174 & 285,2 \\
\hline \multirow[t]{8}{*}{ Norte } & Amapá & 1 & 0,24 & 5 & 0,51 & 4 & 400,0 \\
\hline & Pará & 5 & 1,20 & 26 & 2,64 & 21 & 320,0 \\
\hline & Acre & 1 & 0,24 & 4 & 0,41 & 3 & 300,0 \\
\hline & Rondônia & 4 & 0,96 & 17 & 1,73 & 13 & 275,0 \\
\hline & Tocantins & 4 & 0,96 & 11 & 1,12 & 7 & 150,0 \\
\hline & Amazonas & 6 & 1,45 & 11 & 1,12 & 5 & 100,0 \\
\hline & Roraima & 0 & 0,00 & 4 & 0,41 & 4 & -- \\
\hline & Subtotal & 21 & 5,06 & 78 & 7,93 & 57 & 271,4 \\
\hline \multirow[t]{5}{*}{$\begin{array}{l}\text { Centro- } \\
\text { Oeste }\end{array}$} & Mato Grosso & 5 & 1,20 & 27 & 2,75 & 22 & 440,0 \\
\hline & $\begin{array}{l}\text { Distrito } \\
\text { Federal }\end{array}$ & 7 & 1,69 & 25 & 2,54 & 18 & 257,1 \\
\hline & Goiás & 11 & 2,65 & 36 & 3,66 & 25 & 227,3 \\
\hline & $\begin{array}{l}\text { Mato Grosso } \\
\text { do Sul }\end{array}$ & 6 & 1,45 & 15 & 1,53 & 9 & 150,0 \\
\hline & Subtotal & 29 & 6,99 & 103 & 10,48 & 74 & 255,2 \\
\hline \multirow[t]{5}{*}{ Sudeste } & Rio de Janeiro & 38 & 9,2 & 80 & 8,14 & 42 & 110,5 \\
\hline & Minas Gerais & 62 & 14,94 & 114 & 11,60 & 52 & 83,9 \\
\hline & São Paulo & 109 & 26,27 & 219 & 22,28 & 110 & 100,9 \\
\hline & Espirito Santo & 11 & 2,65 & 17 & 1,73 & 6 & 54,5 \\
\hline & Subtotal & 220 & 53,01 & 430 & 43,7 & 210 & 95,5 \\
\hline \multirow[t]{5}{*}{ Sul } & Paraná & 35 & 8,43 & 56 & 5,70 & 21 & 60,0 \\
\hline & $\begin{array}{l}\text { Rio Grande } \\
\text { do Sul }\end{array}$ & 31 & 7,47 & 53 & 5,39 & 22 & 71,0 \\
\hline & $\begin{array}{l}\text { Santa } \\
\text { Catarina }\end{array}$ & 18 & 4,34 & 28 & 2,85 & 10 & 55,6 \\
\hline & Subtotal & 84 & 20,24 & 137 & 13,94 & 53 & 63,1 \\
\hline & TOTAL & 415 & 100 & 983 & 100 & 568 & 136,9 \\
\hline
\end{tabular}

Fonte: Inep (2017).
O gráfico 1 apresenta a expansão anual dos cursos de enfermagem, desde o ano de 1991 até 2017. Nota-se que há homogeneidade no período, quando se observa a contínua expansão da criação de cursos, mais significativa dos anos de 2004 até 2009, quando foram criados 354 cursos em cinco anos, enquanto criaram-se 98 cursos nos cinco anos entre 2009 até 2015. Após 2015, criaram-se 80 novos cursos no período de um ano, totalizando 178 novos cursos entre 2009 a 2016. Já o período de 1991 até 2004 (13 anos) criaram-se 309 cursos.

Gráfico 1. Cursos de graduação em enfermagem por ano no Brasil, 1991 - 2017.

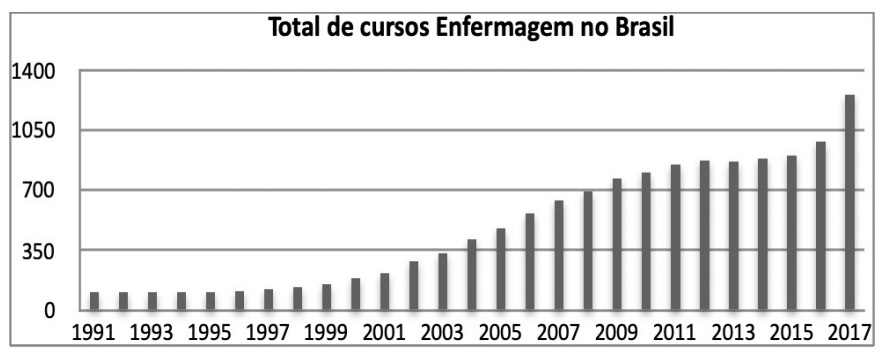

Fonte: Inep (2017)

A tabela 2 traz a distribuição dos cursos de acordo com a sua definição, organização acadêmica e categoria administrativa persistindo denominações como Enfermagem em obstetrícia ou Enfermagem em Saúde Pública, que deveriam ser alterados após 1994 quando o curso passou a ser denominado "enfermagem".

Predominam em 2004, as universidades, seguidas das faculdades. Em 2016 há a expansão das faculdades em $307,8 \%$, maior percentual dentre as outras categorias, e as universidades o menor com 59,7\%. Observa-se um aumento significativo dos centros universitários $(112,9 \%)$,

Destaca-se o crescimento das instituições privadas em doze anos, totalizando $82,6 \%$ do total de cursos enfermagem disponíveis, ofertando 812 cursos no ano de 2016, enquanto as instituições públicas ofertavam 171 cursos. As instituições federais, estaduais e municipais totalizam 17,4\%, mostrando a disparidade entre as instituições de ensino superior, uma vez que o ensino privado em enfermagem expandiu $152,2 \%$ no período, enquanto o público, $83,9 \%$.

Tabela 2. Distribuição dos cursos de graduação em enfermagem no Brasil, de 2004 a 2016. 


\begin{tabular}{llll}
\hline DEFINIÇÃO DO CURSO & & & \\
\hline Definição & 2004 & 2016 & Variação \% \\
\hline Enfermagem & 381 & 974 & 155,6 \\
\hline Enfermagem de saúde pública & 1 & 0 & -- \\
\hline Enfermagem e obstetrícia & 29 & 6 & $(383,3)$ \\
\hline Formação de professor de & & & \\
enfermagem & 4 & 0 & -- \\
Gerontologia & 0 & 2 & -- \\
Obstetrícia & 0 & 1 & -- \\
\hline Total & & & \\
\hline
\end{tabular}

ORGANIZAÇÃO ACADÊMICA

\begin{tabular}{|llll}
\hline Modalidade & 2004 & 2016 & Variação \% \\
\hline Universidade & 238 & 380 & 59,7 \\
\hline Centro Universitário & 62 & 132 & 112,9 \\
\hline Instituto Federal de Ed. Tecnologia & 0 & 2 & -- \\
\hline Faculdade & 115 & 469 & 307,8 \\
\hline Total & 415 & 983 & 136,9
\end{tabular}

Fonte: Inep (2017).

Conforme a tabela 3, até 2004, não havia nenhum curso na modalidade à distância, enquanto na presencial havia 415. Em 2016 havia 100 cursos à distância, com 77.322 mil vagas autorizadas, das quais havia 8.138 matrículas, correspondendo a $10,52 \%$ de vagas preenchidas. Todas as vagas na educação à distância são privadas.

Tabela 3. Cursos de graduação em enfermagem na modalidade presencial e à distância no Brasil de 2004 a 2016.

\begin{tabular}{|llll|}
\hline Modalidade de Ensino & 2004 & 2016 & Variação \% \\
\hline Modalidade & 415 & 883 & 112,8 \\
\hline Graduação presencial & 0 & 100 & -- \\
\hline Graduação à distância & 415 & 983 & 136,9 \\
\hline Total & & & \\
\hline
\end{tabular}

Fonte: Inep (2017).

Vale destacar que a região Norte é a que menor apresenta instituições presenciais, mas está em segundo lugar em maior quantidade de cursos à distância, atrás da região Nordeste à frente do Sudeste. Isso remete ao questionamento de quais fatores estão sendo considerados para a abertura de cursos à distância nessas regiões, tão carentes de cursos presenciais. Pensa-se que essa disseminação de polos na região possa estar relacionada à um acesso mais fácil, especialmente em regiões mais precárias.
A tendência de expansão dos cursos se manteve principalmente nas regiões Nordeste e Norte. No que tange à extinção dos cursos, evidenciou-se a nível nacional, que foram desligados 41 cursos de enfermagem, com maior tendência à extinção nas regiões Sul e Sudeste, onde 32 cursos estão em processo de desligamento enquanto quatro já estavam extintos, em setembro de 2017.

\section{DISCUSSÃO}

A partir dos anos de 1990, a tendência à diversificação do sistema educacional superior não parou de se aprofundar, respaldado na afirmação do fracasso da educação pública pelo seu alto custo, baixa eficiência, distância do mercado. Um ano depois, pelo Decreto no 2.306 de 19 de agosto de 1997, revogado, posteriormente, pelo Decreto no 3.860 de 9 de jutho de 2001, foi definida, para o sistema nacional de ensino, a organização acadêmica das instituições de ensino superior de forma que o sistema universitário vem se transformando rumo a um sistema pós-secundário, amplamente diversificado, com múltiplos arranjos institucionais, influenciados por entidades internacionais, com a criação de novos tipos de instituições de ensino superior (IES), como a universidade especializada e os centros universitários ${ }^{(6)}$.

Em 1995, início do primeiro mandato do presidente Fernando Henrique Cardoso (FHC), registrava-se a oferta de $39,8 \%$ das matrículas de educação superior em instituições públicas e 60,2\% nas privadas. Em 2002, no final de seu mandato, a tendência privatizante intensificou-se, com 30,8\% das matrículas em instituições públicas para $69,2 \%$ nas privadas. Em 2010, no final do segundo governo do presidente Luís Inácio Lula da Silva, o crescimento da rede privada permaneceu como tendência, chegando-se a $25,8 \%$ de matrículas nas IES públicas e $74,2 \%$ nas privadas. Em 2013, quase ao final do primeiro governo de Dilma Rousseff, permaneceu a primazia de matrículas nas IES privadas, mas verifica-se decréscimo passando a 73\%, em função principalmente, da implantação do programa governamental de "Reestruturação e Expansão das Universidades Federais"(7).

$\mathrm{Na}$ graduação em enfermagem destacam-se cursos na rede privada, a partir de 1997, quando existiam 45 cursos privados e, em 2004 eles eram 322, ou seja, um aumento de $837,77 \%$. Em relação à oferta de vagas, em 1991 existiam 3.835, e em 2004 eram 5.597 na rede pública, uma ampliação de $45,99 \%$. Concomitantemente, a rede privada mostrou um aumento de 1.687,66\% de 1991 a 2004, sendo que em 1991 havia 3.625 e, em 2004, 64.803 matrículas $^{(8)}$.

No decênio de 2001 a 2011, foi observada elevação de $393 \%$ do número de cursos nas instituições privadas, e 122\% na rede pública. Nesta comparação, o número de cursos de 
graduação em enfermagem no Brasil, em 2011 era de 826, dos quais 160 em Instituição de Ensino Superior (IES) públicas e 666 em IES privadas. Em 2012, esse número foi de 838 cursos e, em 2013, o número de cursos atingiu $888^{(3,9)}$

A fase que abrangeu os mandatos do presidente Fernando Henrique Cardoso e o início do primeiro mandato do governo Lula é marcada pela edição de inúmeras legislações, que impactaram significativamente as IES, relacionadas à pesquisa aplicada, cursos rápidos, apoiados em novas tecnologias de informação e comunicação; processos avaliativos ou de regulação focados em resultados. Destaca-se a progressiva perda da autonomia universitária, diretamente relacionada à emergência de uma heteronomia de gestão e de uma nova relação entre a universidade e o setor empresarial, que começa a ter curso $^{(10)}$.

A partir de 2003, programas foram implementados para colaborar com a democratização da Educação Superior no país, lançadas, desde o primeiro mandato do presidente Lula, até parte do governo de Dilma Rousseff, programas estes que mudaram as instituições e colaboraram para a expansão do ensino superior brasileiro, como o de Reestruturação e Expansão das Universidades Federais; o incremento à EaD, em especial com a criação do sistema Universidade Aberta do Brasil e a proliferação de cursos "tecnológicos". Quanto à rede privada, a adaptação do Fies (Fundo de Financiamento Estudantil) e a generalização das isenções fiscais, previdenciárias e de renúncia fiscal pelo ProUni foram as principais iniciativas, cobertas com fundo público, que podem ser responsabilizadas pela expansão(10,11).

Neste modelo de expansão, o Estado transferiu a responsabilidade da educação superior para a livre-iniciativa, sob a justificativa de autonomia que, por um lado, favorece a iniciativa privada para a abertura indiscriminada de cursos e vagas, e, por outro, desfavorece a expansão do ensino público por meio da regulação dos recursos financeiros ${ }^{(12)}$. Há carência de estudos avaliativos do impacto deste movimento quanto ao ingresso e permanência de alunos com dificuldades financeiras e que não lograram entrar em instituições públicas. Estudo mostrou dificuldades na vivência universitária, desde a "conquista" da bolsa, vista como benefício do Estado até os preconceitos pela forma de inserção(13).

O aumento dos cursos, principalmente entre os anos de 2004 a 2009, decorre das políticas de governo que, ao passo que expandiram o número de cursos, criaram medidas para oportunizar o ingresso de estudantes em instituições privadas. Se a expansão foi iniciada e ampliada nos governos de Fernando Henrique Cardoso elas ganharam fôlego nos gover- nos de Luiz Inácio Lula da Silva para continuar nos governos da presidente Dilma Rousseff.

A expansão e distribuição de cursos é dispare, pois algumas regiões permanecem com poucos cursos, evidenciado pelo crescimento lento em relação a outras regiões. A região que melhor equilibra a oferta de cursos relacionados à localização é a Centro-Oeste. Nas regiões Norte e Nordeste predomina a concentração de oferta nas capitais, sendo que a região Norte abriga o menor número de IES ${ }^{(14)}$

Em 2010 o cenário era semelhante, com a expansão ocorrendo predominantemente na região Sudeste, que absorvia $43 \%$ do total de cursos de graduação em enfermagem, seguida da região Nordeste, que passou a dispor de $24,12 \%$, a região Sul, com $14,61 \%$, Centro-Oeste com $10,96 \%$, e região Norte, que abrigava $7,3 \%$ dos cursos $^{(15)}$.

Vale destacar que a desigualdade na oferta de cursos se faz presente não apenas entre as regiões, mas também no interior das mesmas. Pode-se citar como exemplo a região Sudeste, que em 2010 contava com 43\% de cursos funcionantes, e desses, 36,7\% estavam no estado de São Paulo. Observa-se uma enorme concentração de investimentos públicos onde a capacidade instalada de recursos humanos qualificados e de infraestrutura preexistentes já é consideravelmente elevada ${ }^{(15)}$

A região Sudeste destaca-se por ser a que mais expandiu, enquanto em último lugar está a região Norte, sendo que o estado que menos obteve ampliação foi Roraima, que partiu da ausência de cursos em 2004 para três cursos em 2015 corroborando com alguns estudos sobre a desproporção inter-regional dos cursos ${ }^{(7,15)}$.

Com esse aumento progressivo dos cursos de enfermagem, principalmente na região Sudeste mostra que a profissão predomina nos centros urbanos, e que pode ser resultado de alguns fatores, tais como as necessidades de saúde da população, relação enfermeiro por habitante e a sustentação do modelo de atenção atual do país. A ampliação do número de vagas e cursos deveria ser acompanhada da ampliação da discussão sobre o seu papel social e de força política para reduzir a precarização do mundo do trabalho(1)

Observou-se aumento das faculdades após o ano de 2004 diretamente ligadas com a expansão do ensino superior privado, não associado a projetos de ensino, pesquisa e extensão e não exigindo professor em tempo integral com mestrado e doutorado. Por isso, pode estar ocorrendo a expansão de ensino dissociada da qualidade, evidenciada pela desobrigação dessas instituições das atividades de pesquisa e extensão e a não exigência de docentes qualificados.

Após a publicação da LDB de 1996(16), que estabeleceu 
as prerrogativas de autonomia dos centros universitários, sem a obrigatoriedade de associação com ensino, pesquisa e extensão, e com menores exigências em termos de percentual de docentes em tempo integral do que as universidades, o interesse entre as IES privadas particulares pela criação e manutenção de universidades diminuiu, enquanto o interesse pela criação e manutenção de centros universitários cresceu, já que as exigências para os centros são menores que para as universidades ${ }^{(17)}$.

Outra estratégia para a expansão da educação superior foi a abertura de cursos à distância. Ao longo dos anos, as instituições à distância públicas e privadas se inseriram de maneira distinta, sendo que até o final de 2002, no governo de FHC, as instituições que ofertavam ensino à distância eram do setor público e, a partir de 2003 houve aumento crescente da participação do setor privado nesta categoria. No ano de 2004, a iniciativa privada ultrapassava o setor público em relação ao número de matrículas em cursos à distância. Em 2010, do total de 748.577 das matrículas (80,48\%) eram provenientes de EaD privadas, enquanto 181.602 eram matrículas (19,52\%) públicas ${ }^{(18)}$

Considerando que a LDB regulamentou a criação de cursos à distância em 1996, dados sobre essa modalidade só aparecem nas estatísticas no ano 2000, mas o curso de enfermagem ainda não havia aderido. Como se observa 100\% das matrículas à distância, em 2016 eram ofertadas em IES privadas, estando as instituições públicas sem nenhuma vaga para o curso(6). A maior concentração de cursos à distância em enfermagem encontra-se na região Nordeste e Norte, no ano de 2017 , com 52,83\%, enquanto as demais regiões contavam com 47,16\%, conforme dados disponibilizados no e-Mec.

As entidades de saúde têm se posicionado contrariamente a educação superior à distância em consonância com a premissa da impossibilidade de se constituir profissionais de saúde em cursos à distância, tema que requer articulação para forçar o seu fechamento ${ }^{(19,20)}$. Visitas in loco identificaram que as condições de oferta da graduação em enfermagem à distância são precárias, sem laboratórios, biblioteca ou condições mínimas de apoio; a maioria dos polos não oferece condições para a prática de estágio supervisionado. As aulas práticas correspondem a 7,79\% da carga horária total dos cursos EaD, contrariando o preconizado pelas Diretrizes Curriculares Nacionais do curso de graduação em Enfermagem ${ }^{(19)}$.

$\mathrm{Na}$ área da saúde, especialmente na formação inicial são imperativos os diálogos entre professores e colegas, para discussão e problematização de diversos temas, saberes e práticas. É crucial que o aluno vivencie a realidade prática e presencial, realizando estágios, vivências clínicas em ambientes reais de cuidado, mediadas pelo professor. A formação em enfermagem deve articular técnica, ciência, ética, relação humana e pensamento crítico e, para tanto, a relação entre os autores do processo ensino aprendizagem (professor, aluno, pessoa cuidada, família, grupos, comunidades) é condição para que a formação seja vivenciada e possa contribuir com a construção de sujeitos comprometidos com o SUS.

Revela-se que a expansão da enfermagem se deu, primordialmente em espaços privados, explicitando o processo de mercantilização do ensino. Isso pode resultar em um ensino descomprometido com as necessidades de saúde, pois o maior investimento não é em qualidade de ensino e capacitação profissional, mas sim em comercializar a educação superior, já que essa evolução também se dá sem a avaliação das necessidades regionais.

\section{Limitações do estudo}

O estudo limita-se por contemplar uma série histórica em tempo reduzido, e por ser exclusivamente quantitativo, não permitindo dimensionar a qualidade do ensino ofertado.

\section{Contribuições para a prática}

Contribui para a divulgação do panorama de expansão dos cursos de graduação em enfermagem; para voltar o olhar para a formação do enfermeiro, no que diz respeito à qualidade de ensino que, necessariamente passa pela qualidade das instituições que ofertam os cursos que pode ser objeto de estudos futuros. Reconhecer como se encontra o cenário da graduação em enfermagem é condição para a organização da categoria e proposição de alternativas.

\section{CONSIDERAÇÕES FINAIS}

Constatou-se que as regiões Norte e Nordeste se destacaram pela expansão nos cursos de graduação em enfermagem, em continuidade à década passada, quando a ampliação dos cursos se deu primordialmente em instituições privadas. Em contrapartida, nas demais regiões foi constatado o fenômeno de extinção e desaceleração da criação dos cursos, sendo evidenciado neste estudo, as regiões Sul e Sudeste.

Todavia, afirma-se que há discrepância da expansão nas diferentes regiões do país, ou seja, o crescimento, em números absolutos é maior na região Sudeste se comparado às outras regiões. Porém, proporcionalmente, as regiões Norte e Nordeste se destacaram pela contínua expansão.

\section{Contribuições dos autores:}

Concepção e/ou desenho, análise e interpretação dos dados: LKM, RMR, RKS. Redação do artigo, revisão final, revisão crítica: LKM, RMR, RKS, SFRC, MSL. 


\section{REFERÊNCIAS}

1. Magalhães SS. et al. Expansão do ensino de enfermagem no Brasil: evidências históricas e perspectivas da prática. Enferm Foco [Internet] 2013 [cited 2017 out 24]; 4(3/4):167-170, 2013. Available from: <http://revista.cofen.gov.br/index.php/enfermagem/article/ view/542/225>.

2. Silva KL, Sena RR, Grillo MJC, Gandra EC, Silveira MR. Expansão dos cursos de Graduação em Enfermagem: dilemas e contradições frente ao mercado de trabalho. Rev Esc Enferm USP. [Internet] 2013 [cited 2017 ago 10]; 47(5):1219-26. Available from: <http://www.scielo.br/pdf/reeusp/v47n5/pt_0080-6234-reeusp-47-05-1211.pdf>

3. Teixeira E, Fernandes JD, Andrade AC, Silva KL, Rocha MEMO, Lima RJO. Panorama dos cursos de Graduação em Enfermagem no Brasi na década das Diretrizes Curriculares Nacionais. Rev Bras Enferm. [Internet] 2013 [cited 18 jan 2018] 66(n. esp):102-10. Available from: <http://www.scielo.br/pdf/reben/v66nspe/v66nspeal4.pdf>

4. Brasil. Instituto Nacional de Estudos e Pesquisas Educacionais Anísio Teixeira (Inep). [Internet] Dados sobre a graduação em enfermagem de 1991 a 2004 [cited 2017 fev 10] Sistema de informação ao Cidadão do Governo Federal, 2017. Available from: <https://esic cgu.gov.br/sistema/site/index.aspx?ReturnUrl=\%2fsistema\%2fPedido\%2fDetalhePedido.aspx\%3fid\%3dJY8dPLsmCBO\%3d\&id=JY$8 \mathrm{dPLsmCBO}=>$.

5. Brasil. Instituto Nacional de Estudos e Pesquisas Educacionais Anísio Teixeira (Inep). Instituições de Educação Superior e Cursos Cadastrados. Sistema E-MEC. [Internet] 2017 [cited 2017 set 11] Consulta avançada. Available from: <<http://emec.mec.gov.br/>

6. Mancebo D, Assis LM, Lima DCBP. Expansão da educação superior: balanço, perspectivas e desafios. Editorial. RBPAE. [Internet] 2016 [cited 2018 fev 11] 32(3):64-652. Available from: <http://seer ufrgs.br/index.php/rbpae/article/view/70261/39674>

7. Mancebo D, Vale AA, Martins TB. Politicas de expansão da educação superior no Brasil 1995-2010. Rev Bras Educ, [Internet] 2015 [cited 2017 ago 17]; 20(60):31-50. Available from: <http://www.scielo. br/pdf/rbedu/v20n60/1413-2478-rbedu-20-60-0031.pdf>

8. Teixeira E, Vale EG, Fernandes JD, De Sordi MRL. Trajetória e tendências dos Cursos de Enfermagem no Brasil. Rev Bras Enferm [Internet] 2006 jul-ago [cited 2018 jan 18] 59(4): 479-87. Available from: <http://www.scielo.br/pdf/reben/v59n4/a02v59n4.pdf>.

9. Cabral IE, Teixeira E, Fernandes JD, Andrade AC, Silva, KL, Rocha MEMO, Lima RJ. Panorama da educação em enfermagem no Brasil. In: Seminário Nacional de Pesquisa em Enfermagem 17, 3 5. Anais. Natal: Associação Brasileira de Enfermagem - Seção Rio Grande do Norte [Internet] 2013 [cited 2017 fev 14]. Available from: <http:// www.abeneventos.com.br/anais_senpe/17senpe/pdf/901lme.pdf>.

10. Mancebo D. Crise político-econômica no Brasil: breve análise da educação superior. Educ. Soc., Campinas [Internet] 2017 [cited 2018
$8 \mathrm{fev}$ ] 38(141): 875-892. Available from: <http://www.scielo.br/pdf/es/ v38n141/1678-4626-es-es0101-73302017176927.pdf>.

11. Barros ASX. Expansão da educação superior no Brasil: limites e possibilidades. Educ. Soc., Campinas. [Internet] 2015 [cited 2018 ago 19] 36(131): 361-390. Available from: <http://www.scielo.br/pdf/es/ v36n131/1678-4626-es-36-131-00361.pdf>

12. Galleguillos TGB, Catani AM. Avaliação da educação superior no Brasil e a expansão da educação superior em enfermagem. Educ. Pesqui., [Internet] 2011 [cited $2018 \mathrm{fev}$ 8]; 37(4): 843-860. Available from: <http://www.scielo.br/pdf/ep/v37n4/allv37n4.pdf>

13. Fontenele TLL, Crisóstomo VL. PROUNI-pontos controversos sob a análise de alunos bolsistas. Avaliação, Campinas [Internet] 2016 [cited 2017 jan 24]: 21(3):739-765. Available from: <http://www.scielo.br/ pdf/aval/v2ln3/1982-5765-aval-21-03-00739.pdf>.

14. Santos CA, Ferreira S. O Plano Nacional de Educação e os desafios da expansão da educação superior. In: SoUZA, J. V. Expansão e Avaliação da Educação Superior Brasileira: formatos, desafios e novas configurações. Belo Horizonte, MG: Fino Traço/Faculdade de Educação da Universidade de Brasilia, p. 55-72.

15. Erdmann AL, Fernandes JD, Teixeira GA. Panorama da educação em enfermagem no Brasil: graduação e pós-graduação. Enferm foco [Internet] 2011 [cited 2017 ago 30]; 2(supl):89-93. Available from: <http://revista.cofen.gov.br/index.php/enfermagem/article/ view/91/76>

16. Brasil. Presidência da República. Subchefia para assuntos juridicos. [Internet] Lei no 9.394, de 20 de dezembro de 1996. Estabelece as diretrizes e bases da educação nacional. [cited 2017 jan 22]. Brasilia, DF, 1996. Available from: <http://www.planalto.gov.br/ccivil_03/leis/ L9394.htm>.

17. Sguissardi V. Estudo diagnóstico da política de expansão da (e acesso à) educação superior no Brasil 2002 - 2012. 2014. mimeo.

18. Mancebo D, Martins TB. Expansão do ensino à distância: pressupostos para sua análise e marcos regulatórios. In: Mancebo D. Silva júnior JR. Trabalho docente e expansão da educação superior brasileira. Rio de Janeiro: EdUERJ, 2012, p. 117-146.

19. Brasil. Conselho Nacional de Saúde (CNS). [Internet] Resolução № 515, de 07 de outubro de 2016. [cited 2017 set 22] Brasilia, 2016. Available from: <http://conselho.saude.gov.br/resolucoes/2016/ Reso515.pdf>.

20. Cofen. Conselho Federal de Enfermagem. Cofen propõe projeto de lei proibindo EaD na Enfermagem, 2015. Available from: <http:// www.cofen.gov.br/cofen-propoe-projeto-de-lei-proibindo-ead-na-enfermagem_34001.html>.

RECEBIDO: 03/06/2019

ACEITO: 08/07/2019 